\title{
REMARKS ON MODULE-FINITE PAIRS
}

\author{
by JAMES A. HUCKABA and IRA J. PAPICK
}

(Received 24th March 1980)

Let $R \subsetneq T$ be an extension of commutative rings having the same identity. A. Wadsworth (10) studies the situation when $R$ and $T$ are integral domains, and all rings between $R$ and $T$ are Noetherian. In this case $(R, T)$ is called a Noetherian pair. In a similar vein, E. Davis (4) studies normal pairs and I. Papick (8) shows when coherent pairs are Noetherian pairs.

These papers are the motivation for this article. We study the concept of a module-finite pair-that is a pair $(R, T)$ in which each intermediate ring (including $T$ ) is module-finite over $R$. It is clear that in the category of Noetherian rings such pairs abound, and it is our intention (Theorem 2) to show that in a very general setting this is the only place these pairs exist. However, in complete generality, module-finite pairs do exist in non-Noetherian categories. (Remark (a)). Note that we do not necessarily assume that $R$ and $T$ are domains.

Let $(R, T)$ be a module-finite pair, $I=\left(R_{\dot{R}} T\right), P \in \operatorname{Spec}(R)$, and $S=R+P T$. By integrality, $P T \in \operatorname{Spec}(S)$. With this notation we have:

Lemma 1. If $I \subseteq P$, then $(S: T)=P T$.

Proof. For notational purposes, let sub $U$ denote all localizations at $R \backslash P$; e.g., $S_{U}=S_{R \backslash P}$ and $P_{U}=P R_{P}$. By the ring theory version of (7, Exercise 41(c), p. 46) $R_{U} \subsetneq T_{U}$, and so $\left(R_{U}, T_{U}\right)$ is a proper module-finite pair. Hence by Nakayama's Lemma $R_{U}+P_{U} T_{U} \neq T_{U}$. But $S_{U}=R_{U}+P_{U} T_{U}$, and so we may choose a $t \in T_{U} \backslash S_{U}$. Notice that $\left(S_{U}: t\right)=P_{U} T_{U}$, since $S_{U} / P_{U} T_{U}$ is a field. Hence,

$$
P_{U} T_{U}=\left(S_{U_{S_{U}}}: T_{U}\right)=\left(S_{\dot{S}} T\right)_{U}(1, \text { Corollary } 3.15)
$$

From this it follows that $P T \supset\left(S_{\dot{S}} T\right)$. Therefore $P T=\left(S_{\dot{S}} T\right)$ and the proof is now complete.

Theorem 2. Let $(R, T)$ be a module-finite pair with $R$ an integral domain, and set $I=(R: T)$.

(a) If $I=(0)$, then $R$ is a Noetherian domain.

(b) If $I \neq(0), T$ a domain, and $R$ is a coherent domain, then each ideal of $R$ containing $I$ is finitely generated. In particular, $R / I$ is a Noetherian ring. 
Proof. (a) The proof of this may be found in (8, p. 561). However, we include it here for completeness. Let $J$ be an ideal of $R$. Since $T$ is not contained in the quotient field of $R$, we may choose an element $t \in T \backslash R$ such that $R[t]$ is a free $R$-module with basis $\left\{1, t, \ldots, t^{n-1}\right\}, n \geqq 2$. Let $S=R+J t+J^{2} t^{2}+\ldots+J^{n-1} t^{n-1}$. Observe that $S$ is a subring of $T$, and moreover, $S=R \oplus J t \oplus J^{2} t^{2} \oplus \ldots \oplus J^{n-1} t^{n-1}$. As $S$ is module-finite over $R$, so is $J$ module-finite over $R$. Hence, $J$ is a finitely generated ideal of $R$.

(b) To show that each ideal of $R$ containing $I$ is finitely generated, it suffices to show that each prime ideal of $R$ containing $I$ is finitely generated (7, Exercise 24, p. 65).

Let $P \in \operatorname{Spec}(R)$ such that $I \subseteq P$, and set $S=R+P T$. Note that since $I \neq(0), T$ is a domain contained in the quotient field of $R$. Hence, as $R$ is coherent and $R \subseteq S$ is a module-finite extension of domains, then $S$ coherent (6, Corollary 1.5). By Lemma 1 , $P T=(S: T)$. Then since $T=\sum_{i=1}^{m} S\left(a_{i} / b_{i}\right)$ for some $a_{i}, b_{i} \in S$, we have $(S: T)=\bigcap_{i=1}^{m}\left(b_{i s}: a_{i}\right)$; and so the coherence of $S$ forces $P T$ to be finitely generated in $S$ (3, Theorem 2.2). Finally, as $P T \cap R=P$, it follows that $P$ is finitely generated in $R$ (2, Exercise $11(\mathrm{~d}), \mathrm{p}$. 44). The proof of Theorem 2 is complete.

Let $\bar{R}$ (resp., $\bar{T}$ ) denote $R / I$ (resp., $T / I$ ) where $I=\left(R_{R}: T\right)$.

Lemma 3. Let $T$ be a ring extension of $R$. Then $(R, T)$ is a module-finite pair if and only if $(\bar{R}, \bar{T})$ is a module-finite pair.

Proof. $(\rightarrow)$ : Clear.

$(\leftarrow)$ : Let $R \subset S \subset T$ where $S$ is a subring of $T$. Since $I$ is an ideal of $S, \bar{R} \subset \bar{S} \subset \bar{T}$ where $S / I=\bar{S}$. Write $\bar{S}=\sum_{i=1}^{n} \bar{R} \bar{s}_{i}$ for some $s_{i} \in S$. Then

$$
S \subset \sum R s_{i}+I \subset \sum R s_{i}+R 1 \subseteq S .
$$

Hence, $S=R s_{1}+\ldots+R s_{n}+R 1$.

A version of Theorem 2(a) can now be established for rings with zero divisors.

Proposition 4. Let $(R, T)$ be a module-finite pair and assume that $I=\left(R_{i} T\right)=(0)$. Then $R / P$ is a Noetherian domain for each prime ideal $P$ of $R$.

Proof. For a fixed $P \in \operatorname{Spec}(R)$, let $S=R+P T$. By Lemma $1(S: T)=P T$, and by Lemma $3(S / P T, T / P T)$ is a module-finite pair. The conductor of $S / P T$ in $T / P T$ is $(0)$, and $S / P T$ is an integral domain. Applying Theorem 2(a) we see that $S / P T \cong R / P$ is a Noetherian domain.

If the ring $R$ of Proposition 4 has only finitely many minimal prime divisors of (0), then $R / N(R)$ is a Noetherian ring $(N(R)=$ nilradical of $R)$. Thus, if $R$ is also reduced, then it is Noetherian.

\section{Remarks}

(a) There exist "non-coherent" module-finite pairs. For a particular example, let $k \subsetneq K$ be an extension of fields such that $[K: k]<\infty$. Let $V$ be a valuation ring of the form 
$K+M$ such that $M$ is not finitely generated. Then, $R=k+M$ is not coherent (5, Theorem 3), but $(R, V)$ is a module-finite pair. With respect to statement (b) of Theorem 2, it is interesting to note that $R /\left(R_{\dot{R}} V\right)$ is trivially Noetherian, yet $\left(R_{\dot{R}} V\right)=M$ is not finitely generated.

(b) In an attempt to determine general contexts for which the proof of Theorem 2(a) applies, we are naturally led to the following question: If $R \subseteq T$ is a module-finite extension of commutative rings and $\left(R_{R} T\right)=(0)$, then does there exist an element $t \in T \backslash R$ such that $R[t]$ is a free $R$-module with basis $\left\{1, t, \ldots, t^{n-1}\right\}, n \geqq 2$ ? In general the answer is no, and we are thankful to Wolmer $V$. Vasconcelos for suggesting an appropriate counterexample.

Let $(R, M)$ be a local non-Noetherian ring such that $M$ is finitely generated, $M=Z(R)$ (zero divisors of $R$ ), and $\operatorname{Ann}_{R}(M)=(0)(9$, Section 3). Set $T=R(+) M$, the idealization of $M$ in $R$ (7, Exercise 7, p. 63). We claim that $R \subsetneq T$ is the desired example. It is straightforward to see that $T$ is module-finite over $R$, since $M$ is finitely generated, and that $\left(R_{R}: T\right)=(0)$, since $\operatorname{Ann}_{R}(M)=(0)$. To complete the proof of the claim we will show that there does not exist a finitely generated free $R$-module $S$, such that $S$ is a ring and $R \subsetneq S \subseteq T$. Assume such an $S$ exists, and let $1, \alpha$ be distinct $R$-linearly independent elements of $S$. Note that $\alpha=(r, m)$ for some $r \in R, m \in M$. Choose $0 \neq b \in R$ such that $b m=0$, and observe that $(b r) 1-b \alpha=0$, which is a contradiction.

We wish to thank the referee for several helpful suggestions concerning this paper.

\section{REFERENCES}

(1) M. Atryah and I. MacDonald, Introduction to Commutative Algebra (Addison-Wesley, Reading, Mass., 1969).

(2) N. BourbakI, Commutative Algebra (Addison-Wesley, Reading, Mass., 1972).

(3) S. Chase, Direct products of modules, Trans. Amer. Math. Soc. 97 (1960), 457-519.

(4) E. Davis, Overrings of commutative rings III; Normal pairs, Trans. Amer. Math. Soc. 182 (1973), 175-185.

(5) D. Dobss and I. PaPick, When is $D+M$ coherent?, Proc. Amer. Math. Soc. 56 (1976), 51-54.

(6) M. Harris, Some results on coherent rings, Proc. Amer. Math. Soc. 17 (1966), 474-479.

(7) I. KaplansKY, Commutative rings (Allyn and Bacon, Boston, Mass., 1970).

(8) I. PAPICK, When coherent pairs are Noetherian pairs, Houston J. Math. 5 (1979), 559-564.

(9) W. Vasconcelos, Annihilators of modules with a finite free resolution, Proc. Amer. Math. Soc. 29 (1971), 440-442.

(10) A. Wadsworth, Pairs of domains where all intermediate domains are Noetherian, Trans. Amer. Math. Soc. 195 (1974), 201-211.

Department of Mathematics

UNTVERSITY OF MISSOURI

Columbia, Missouri 65211 